\title{
Sphere-to-plane electrodes configuration of positive and negative plasma corona discharge
}

\author{
Sabah I. Wais, Dunia D. Giliyana \\ Department of Physics, Faculty of Sciences, University of Duhok, Kurdistan Region, Iraq \\ Email address: \\ sabahwais@uod.ac (S. I. Wais), duniagiliyana@uod.ac (D. D. Giliyana)
}

To cite this article:

Sabah I. Wais, Dunia D. Giliyana. Sphere-to-Plane Electrodes Configuration of Positive and Negative Plasma Corona Discharge, American Journal of Modern Physics. Vol. 2, No. 2, 2013, pp. 46-52. doi: 10.11648/j.ajmp.20130202.12

\begin{abstract}
An experimental investigation has been carried out with sphere-to-plane geometrical electrodes for positive and negative corona discharge at atmospheric pressure and different inter-electrode separation. The experimental results have been compared particularly with those refer to point-to-plane and point-to-ring geometriesand a similar common feature has been provided. The current and voltage data was subjected to the relation of Townsend and Ferreira and showed one an upward curvature at low applied voltage (low field region) and then a linear behavior was found in high field region. This means that all acquired current-voltage data for both positive and negative coronas confirm Townsend relation at the inter-electrode distance used in this work. The extrapolated corona inception voltage was followed a non-linear tend with electrode gap distance S. The extrapolated potential Vo for positive corona was more sensitive on the distance $\mathrm{S}$ than that of negative corona. The dimensional constants $\mathrm{A}$ and $\mathrm{K}$ were found to be proportional inversely to the electrode gap distance by different power exponent. The ratios between the dimensional constants $\mathrm{A}$ and $\mathrm{K}$ for positive and negative corona approached a constant value at gap distance $(\mathrm{S} \geq 2.5$ $\mathrm{cm})$.
\end{abstract}

Keywords: Current-Voltage Characteristics, Corona Inception Voltage, Electrode Gap Distance, Townsend Relation

\section{Introduction}

Corona discharge has been extensively used in different applications such as electrostatic painting, electret production, electrophotography, etc. [1]. The current that usually carried out by corona discharge flows from a high potential electrode and ionizes the working neutral fluid to create plasma around the electrode. This happens, when the geometrical configuration of the electrodes include an electrode of sharp tip such as point-to-plane, point-to-grade, sphere-to-plane and wire cylinder electrodes. If the potential of the sharp (tip) electrode is large enough, the fluid at tip point ionizes and becomes conductive leading to the formation of highly curved region on electrodes. Corona discharge is therefore associated with non-uniform electric field. The type of corona is determined by the polarity of the highly curved tip electrode. If the voltage of the tip is positive with respect to the plane electrode, the corona is said to be positive corona while it is called negative corona when the tip is in negative voltage [2].

The current-voltage characteristics of corona discharge for positive and negative polarities are strikingly different because of the mass different between electrons and charge ions. The current-voltage characteristics in corona discharge have been described by several empirical formulas involving different geometrical configuration of electrodes. Corona discharge of point-toplane electrodes has been studied at first by Rontgen at the end of $19^{\text {th }}$ century [1]. Townsend derived a formula to describe the behavior of current-voltage characteristics of dc steady corona for coaxial cylindrical geometry. Henson [3] declared by an empirical formula that the Townsend relationship could also be used for point-toplane geometry as: 


$$
I=A V\left(V-V_{o}\right)
$$

where Vo is the corona inception voltage and $\mathrm{A}$ is a dimensional constant which is depended on the interelectrode separation and the geometrical configuration of the electrodes. Equation (1) was later modified by Ferreira [1] as:

$$
I=K\left(V-V_{o}\right)^{2}
$$

where $\mathrm{K}$ is a constant which is determined experimentally. An experimental investigation of the current voltage characteristics for positive and negative corona has been demonstrated by Xiangbo[4]with an empirical formula that met with some physical difficulties in explaining the results. Finally, a more specific form is expressed as follows:

$$
I=C_{1} S^{-0.41}\left(V-C_{2} S^{0.51}\right)^{1.6}
$$

where $\mathrm{C}_{1}$ and $\mathrm{C}_{2}$ are coefficients which depend on electrode geometry and ambient conditions. It is clear from equation (3) that $\mathrm{K}$ and $\mathrm{V}_{\mathrm{o}}$ are proportional to inter-electrode separation $\mathrm{S}$ as: $\mathrm{K} \propto \mathrm{S}^{-0.41}$ and $\mathrm{V}_{\mathrm{o}} \propto \mathrm{S}^{0.51}$.

In 2004, Yamada modified the Townsend relationship with a point-to-grid electrode geometry under the influence of ambient temperature and inter-electrode separation [5]. A series of measurements carried out by Ferreira [1] with point-to-plane symmetry has shown that positive corona confirms the Townsend relation while for negative corona this happens only at large point-toplane distances. With point-to-ring geometry, Giubbilini [6] compared the experimental results with the ones that refer to point-to-plane corona. The comparison has provided evidence of the similar feature between point-toring and point-to-plane corona. Several empirical formulas of a common feature and different experimental works were disclosed the validity of the Townsend relationship in describing the current-voltage characteristics of corona discharge for both polarities.

The aim of the present work is to give a detailed description of corona mechanism for positive and negative polarities of sphere-to-plane geometrical electrodes. The acquired current and voltage data is devoted to explore the influence of the inter-electrode separation on corona current, corona inception voltage and the dimensional constants for both polarities. All the experimental measurements were compared with works referred to discharge systems of point-to-plane and point-to-ring geometrical electrodes. The comparison showed that both polarities of sphere-to-plane system confirm Townsend relation. The variation of the ratio of the dimensional constants $\mathrm{A}^{-} / \mathrm{A}^{+}$of equation (1) and $\mathrm{K}^{-}$ $/ \mathrm{K}^{+}$ofequation (2) on the inter-electrode separation were explain and showed an agreement with previous works.

\section{Experimental Setup}

The experimental setup is shown in figure 1; it consists of a brass sphere of diameter $0.85 \mathrm{~cm}$ and flat plane electrode of $4.5 \mathrm{~cm}$ diameter. The voltage is supplied by a dc power supply $\mathrm{V}$ of $(0 \pm 20 \mathrm{Kv})$ connected to the sphere electrode and microammeter A placed between the flat plane electrode and the ground. The sphere is placed on the axis that passes through the center of the plane electrode at different inter-electrode separation $\mathrm{S}$ ranging from 0.5 to $3.0 \mathrm{~cm}$. Data acquisition system is built and used to acquire the current and voltage data. All the measurements were carried out with positive and negative corona at room temperature $27^{\circ} \mathrm{C}$, atmospheric pressure $760 \mathrm{~mm} \mathrm{Hg}$ and relative humidity of about $55 \%$.

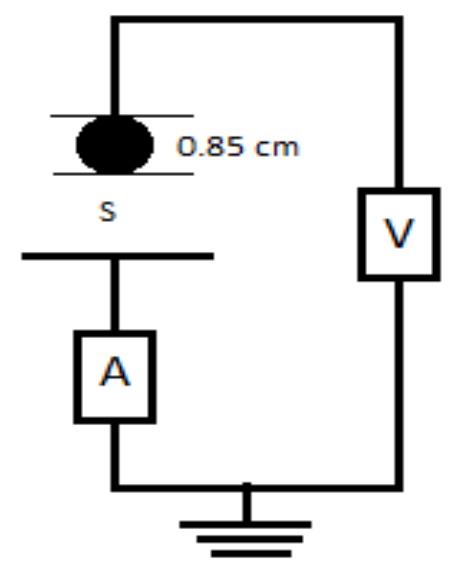

Figure 1.Schematic of experimental setup.

However, two important points are considered in the present work. First, the influence of the diameter of sphere on the corona current was not considered. Second, the currents and voltages will be given in their absolute values and the polarity will be always mentioned to avoid confusion.

\section{Results and Discussion}

This section explores the experimental results of the present work and expresses the influence of interelectrode separation of the sphere plane gap configuration as well as the physical description of positive and negative corona discharge of the concerned gap. This study covered the effect of the gap separation on the corona current, corona inception voltage and the dimensional constants $\mathrm{A}$ and $\mathrm{K}$.

In this work, the corona current with sphere-to-plane is considered for both polarities. This consideration is based on the possibilities of providing different point sources of ions in air depending on the curvature of the 
sphere in front of the plane electrode. The experimental results were compared with the results of recent works regarding point-to-planeand point-to-ring geometries. All the experimental measurements with positive and negative corona were carries out in the regular Trichel pulses regime.

\subsection{Current-Voltage Characteristics}

The current-voltage data that were acquired for positive and negative corona at different inter-electrode separation are summerised in figure 2. As shown, the corona current decreases as the gap separation between the electrode increases. This can be attributted to the low ionization rate near stressed electrode (sphere) and low charge mobility in the drift region. As the discharge has a positive resistance at a fixed pressure with increasing $\mathrm{S}$, the breakdown voltage (that is, corona inception voltage $V_{o}$ ) goes up for negative corona more than that for positive corona as the gap distance $\mathrm{S}$ increased, thus reducing the current produced by the discharge [7].

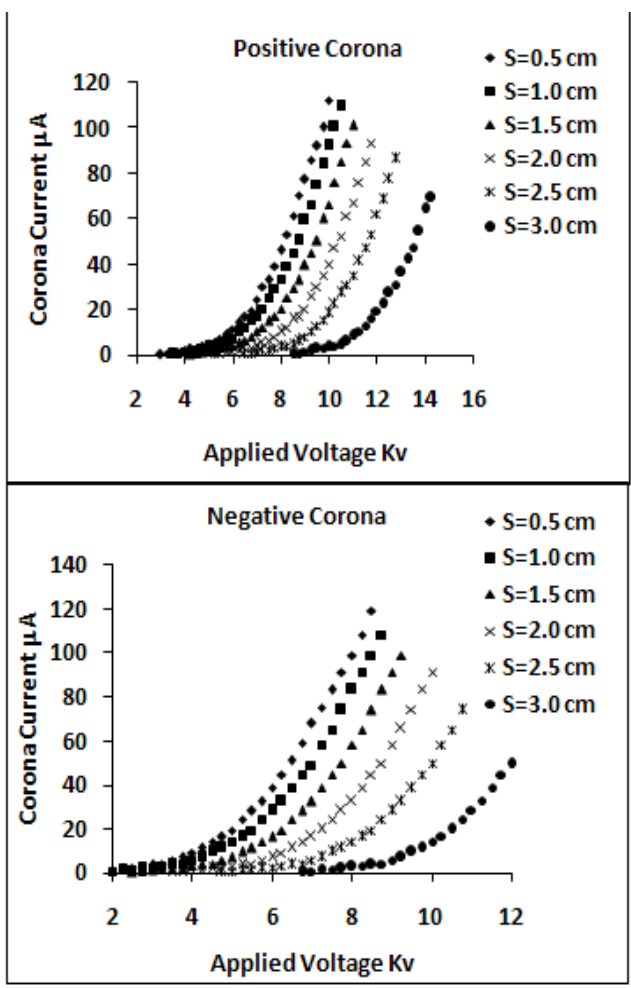

Figure 2. Current-voltage characteristics for positive and negative corona discharge.

The acquired data of figure 2 have been examined for positive and negative corona with the two literature empirical formulae given in equations (1) and (2) as shown in figures 3 and 4 respectively.
As a result of examining the experimental data of the present work with the formula that given by equations (1)and (2) as shown in figures 3 and 4 for both positive and negative coronas, it is indicated that although the two empirical equations do indeed show first approximation agreement with experiment, it is difficult to state that all data are in perfect better agreement with one particular formula or another. A linear behavior is observed in all acquired data for $\mathrm{I}^{1 / 2}$ against $\mathrm{V}$, while $\mathrm{I} / \mathrm{V}$ vs $\mathrm{V}$ one showed an upward curvature. A departure from the linear dependence is also observed in $\mathrm{I}^{1 / 2}$ vs $\mathrm{V}$ at lower applied voltage where the current itself is not very stable. Good straight lines are obtained in all cases of figures 3 and 4; this verifies that equation (1) and (2) are held for all the concerned inter-electrode separation of the present work.

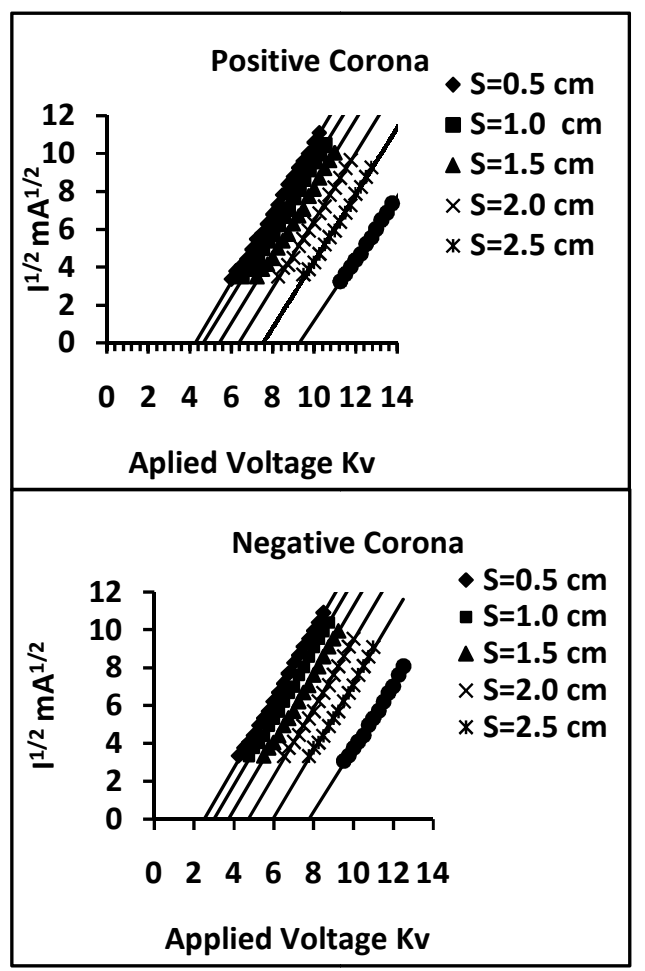

Figure 3. The examining of the experimental data of the present work by applying equation (2): II/2 as a function of the applied voltage $V$.

The upward trend in the characteristics of $\mathrm{I} / \mathrm{V}$ and $\mathrm{I}^{1 / 2}$ versus $\mathrm{V}$ as shown in figures 3 and 4 was explained by an increasing participation of the electronic component on the total corona current. Sigmond [8] deduces an upper characteristic I/V vs V called a space charge saturated one giving the maximum current that could be expected at each voltage from unipolar ionic conduction. The corona current measured in the present work for both polarities cross the Sigmond saturation curve at short inter-electrode distance $\mathrm{S}$ and becoming larger at 
higher voltage and thus clearly indicating an electronic contribution [1].

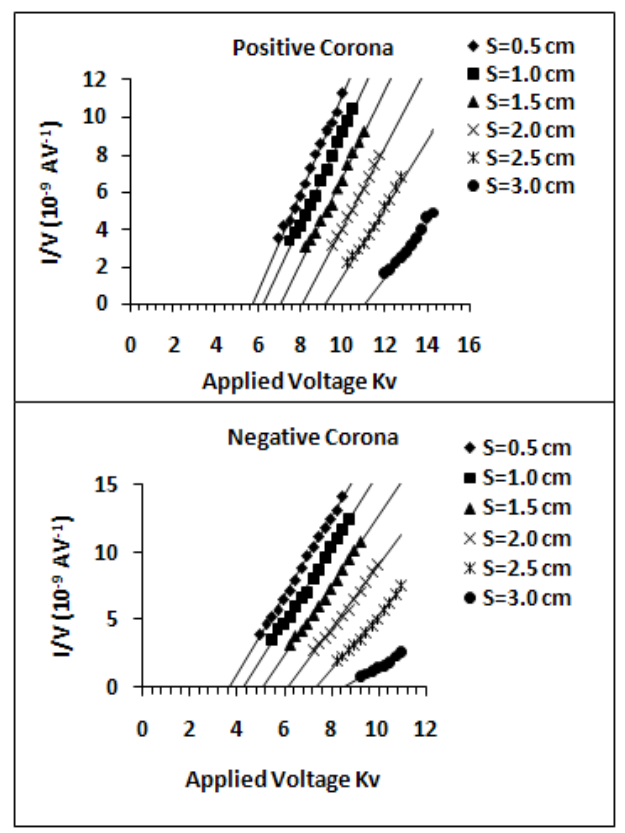

Figure 4. The examining of the experimental data of the present work by applying equation (1): $I / V$ as a function of the applied voltage $V$.

\subsection{The Variation of Corona Inception Voltage $V_{o}$ with $S$}

The corona inception voltage $\mathrm{V}_{\mathrm{o}}$ refers to the starting point at which the corona discharge takes place when the applied voltage exceeds a threshold point. This voltage is essentially independent of the corona current and applied voltage but it is strongly depends on the tip radius of the sharp point electrode and inter-electrode separation $[4,9]$. The corona inception voltage $V_{o}$ for positive and negative corona have been obtained from figure 4, extrapolated the straight lines to the voltage axis. Figure 5 shows that for a given inception current, the resulting corona inception voltage follows a nonlinear trend with the electrode gap separation.

According to figure 5, the corona inception voltage at fixed tip radius is proportional to $\mathrm{S}^{\mathrm{m}}$ (nonlinearly) as follows:

$$
V_{o} \propto S^{m} \rightarrow V_{o}=a S^{m}
$$

This equation can be transformed to a linear form by taking the algorithm of both sides, as follows:

$$
\log _{10}\left(V_{o}\right)=m \log (S)+C_{1}
$$

The parameter $\mathrm{m}$ in equation (5) is determined by the slope of the fitting lines and presents a linear depen- dence on the inert-electrode separation $\mathrm{S}$ as shown in figure 6 . The dimensional constant $\mathrm{C}_{1}$ which is equal to $\log (a)$ is determined from the intercept with $\log \left(V_{o}\right)$, where a is also another dimensional constant as shown in equation 4 . It is clear from figure 6 that the values of the exponent $\mathrm{m}$ are 0.41 and 0.54 for positive and negative corona respectively. The value of $\mathrm{m}$ is determined from a curve fitting method.

It is noted from figure 5 that the extrapolated potential $\mathrm{V}_{\mathrm{o}}$ for positive corona is more sensitive on the distance $\mathrm{S}$ than the extrapolated potential of negative corona. These facts have been also observed in point-toplane corona [4].

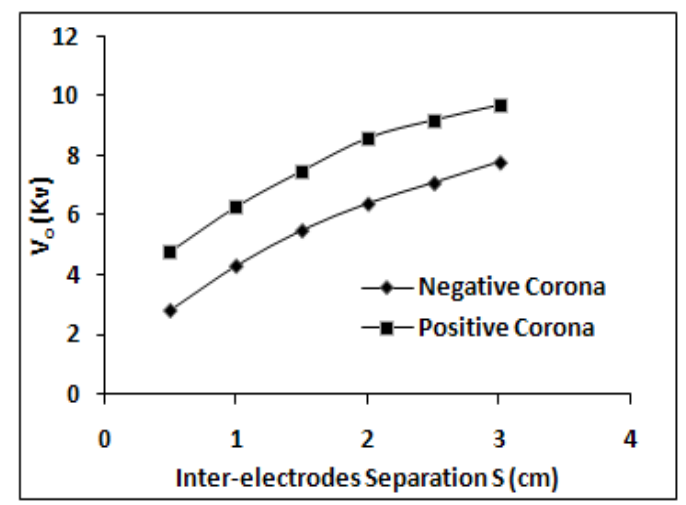

Figure 5. The extrapolated corona inception voltage for positive and negative corona as a function of electrode separation $S$.

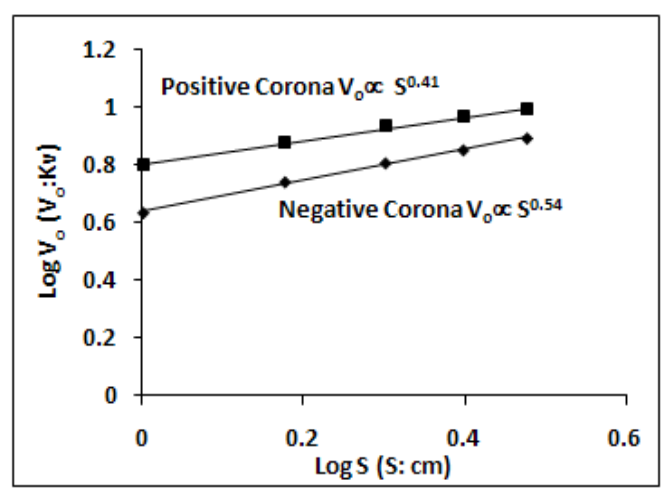

Figure 6. A plot of $\log (\operatorname{Vo})$ vs $\log (S)$. The solid line is obtained by least square fitting.

Moreover, as a result of comparison between the coronas of sphere-to-plane and point-to-plane, a good agreement was observed with Xiangbo [4] in the behavior of corona inception voltage as a function of interelectrode separation where it tended to have a nonlinear variation. Whereas, a significant departure from the results of Ferreira [1] and Giubbilini [6 ] were noted because of the linear behavior of $\mathrm{V}_{\mathrm{o}}$ versus $\mathrm{S}$ that has been dominated in their work. 


\subsection{The Variation of Dimensional Constants aand $K$ with $S$}

The influence of the inter-electrode separation on the dimensional constants $\mathrm{A}$ and $\mathrm{K}$ given in equations (1) and (2) for positive and negative corona is shown in figures 7 and 8 . It is observed an inversely proportional of both $\mathrm{A}$ and $\mathrm{K}$ with electrode gap distance $\mathrm{S}$ and disclosed a significant sensitivity of $\mathrm{K}$ more than A against $\mathrm{S}$. Accordingly, the variation of $\mathrm{A}$ and $\mathrm{K}$ with $\mathrm{S}$ can be represented mathematically as follows:

$$
\begin{aligned}
& A \propto S^{-x} \rightarrow A=b S^{-x} \\
& K \propto S^{-y} \rightarrow K=c S^{-y}
\end{aligned}
$$

where $\mathrm{b}$ and $\mathrm{c}$ are the constants of proportionality. The values of the exponent $\mathrm{x}$ and $\mathrm{y}$ have been determined from the slope fitting lines as referred by figure 7 and 8 . The values of dimensional constants A depend on geometrical factors such as inter-electrodes separation, tip radius of the sharp electrode, the mobility and the permittivity of the vacuum $[5,6]$.

The values of Ahave been deduced from figure 4 for positive and negative corona; these experimental values are plotted in figure 7 as a function of distance $S$ and illustrated a linear dependence of A for both positive and negative coronas on such distance $\mathrm{S}$. It was found that $\mathrm{A}$ is inversely proportional to electrode gap distance $\mathrm{S}$ as $\mathrm{A} \propto \mathrm{S}^{-1.54}$ for positive corona, while it was $A \propto \mathrm{S}^{-2.15}$ for negative corona.

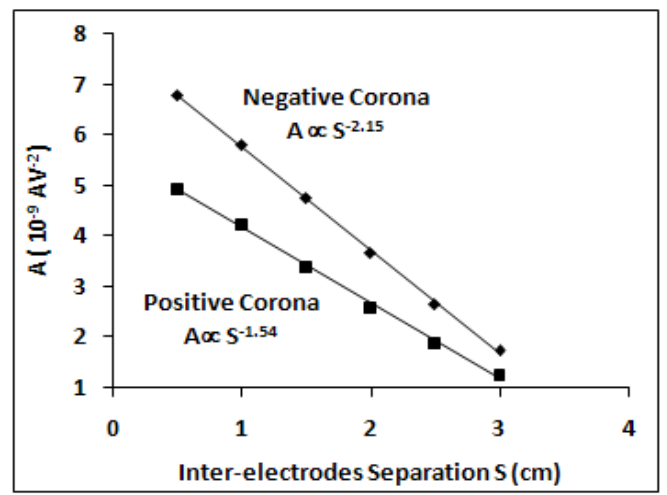

Figure 7. The dependence of the dimensional constant $A$ on the electrode gap distance $S: A \propto S-1.54$ for positive corona and $A \propto S-2.15$ for negative corona. The solid line is obtained by least square fitting.

From the results of figures 4 and 7, it can be inferred that, the current-voltage characteristics confirm Townsend relation, equation (1), where $\mathrm{A}$ and $\mathrm{V}_{\mathrm{o}}$ are experimentally determined constants. For sphere-to-plane geometry, the current-voltage characteristics described by equation (1) are valid for the experimental data of the present work and follow the relation:

$$
I=A^{ \pm} V\left(V-V_{o}^{ \pm}\right)
$$

for positive and negative coronas. Equation (8) was already verified for the current-voltage characteristics in point-to-plane and point-to-ring corona discharge $[1,6]$. This indicates a signification agreement between the present work with those have been done by Ferreira [1] and Giubbilini [6]. Therefore, an experimental analogy should be considered between sphere-to-plane geometry and point-to plane and point-to-ring systems.

Similarly, the experimental values of $\mathrm{K}$ determined from figure 3 are plotted against $\mathrm{S}$ as shown in figure 8 . A linear behavior is also observed for both positive and negative corona but with different values of exponent $\mathrm{x}$ and $y$. According to the results of experimental values of $\mathrm{K}$, the current-voltage characteristics given by equation (2) for sphere-to-plane system can be rewrite for both positive and negative corona as:

$$
I=K^{ \pm}\left(V-V_{o}^{ \pm}\right)^{2}
$$

The constants $\mathrm{K}^{+}$(positive corona) and $\mathrm{K}^{-}$(negative corona) also depended on some geometrical parameters. It was noted from figures 8 , that $\mathrm{K}^{+}$and $\mathrm{K}^{-}$showed a different dependence on the distance $\mathrm{S}$ and was $\mathrm{K}^{+} \propto \mathrm{S}^{-}$ ${ }^{1.57}$ for positive corona and $\mathrm{K}^{-} \propto \mathrm{S}^{-2.25}$ for negative corona

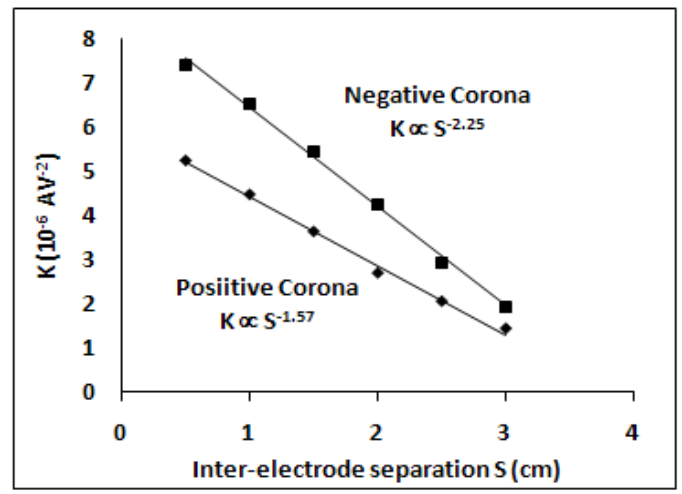

Figure 8. The dependence of the dimensional constant $K$ on the electrode gap distance $S: K \propto S-1.57$ for positive corona and $K \propto S-2.25$ for negative corona. The solid line is obtained by least square fitting.

A similar variation of the dimensional constants A and $\mathrm{K}$ for positive and negative corona have been observed in point-to-plane and point-to-ring coronas. Another common feature between the results of the present work with Ferreira [1] and Giubbilini [6] is that $\mathrm{A}$ and $\mathrm{K}$ for negative corona has higher values than that for positive one, as is apparent from figures 7 and 8 . Therefore, an evidence of the similar feature has been provided by the present work between the sphere-toplane geometry and point-to-plane and point-to ring 
coronas concerning current-voltage characteristics and dependence of the extrapolated inception voltage $V_{o}$ and dimensional constants $\mathrm{A}$ and $\mathrm{K}$ on the distance $\mathrm{S}$.

\subsection{The Variation of $A^{-} / A^{+}$and $K^{-} / K^{+}$with $S$}

All the measurements that have been carried out in the present work have already shown, the Townsend relation holds the current-voltage characteristics for both polarities of sphere-to-plane corona at the concerned gap distances. In order to recognize between positive and negative corona for sphere-to-plane geometry as referred by Ferreira [1], it is also carried out a new measurements with inter-electrode separation going up to $7 \mathrm{~cm}$. the results are summarized in figure 9 where both ratios $\mathrm{A}^{-} / \mathrm{A}^{+}$and $\mathrm{K}^{-} / \mathrm{K}^{+}$are plotted against electrode gap distance $\mathrm{S}$. it was seen that $\mathrm{A}^{-} / \mathrm{A}^{+}$approaches a constant value $(\sim 0.7)$ at distances higher than $2.5 \mathrm{~cm}$, while $\mathrm{K}^{-} / \mathrm{K}^{+}$approaches $(\sim 0.64)$ after the same distances.

As shown in figure 9, the ratios $\mathrm{A}^{-} / \mathrm{A}^{+}$and $\mathrm{K}^{-} / \mathrm{K}^{+}$approached a constant value at gap distance $(\mathrm{S} \geq 2.5 \mathrm{~cm})$. This means that equations (1) and (2) are held both polarities of sphere-to-plane corona and disclosed that different relations hold between current-voltage characteristics for positive and negative corona for short distances $(\mathrm{S}<2.5 \mathrm{~cm})$

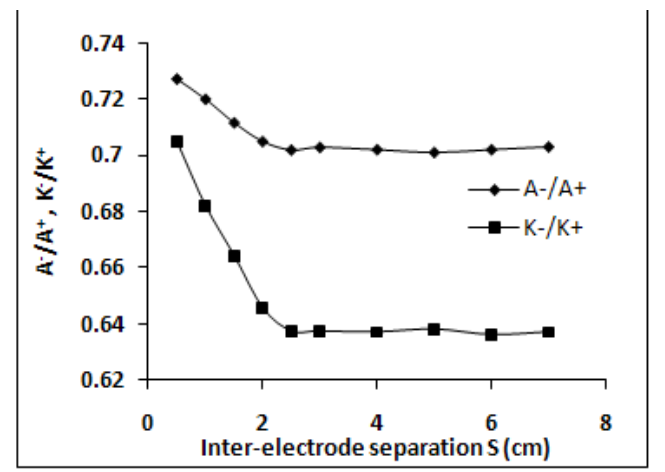

Figure 9. The variation of the ratios $A-/ A+$ and $K-/ K+$ versus $S$.

However, the larger the distance S, the smaller contribution of the electronic component and therefore, the current -voltage corona characteristics for each polarity should become Townsend type, as indeed observed in this work. This would indicate, a decreasing of electronic contribution should make the $\mathrm{A}^{-} / \mathrm{A}^{+}$and $\mathrm{K}^{-} / \mathrm{K}^{+}$ratios approach the theoretical relation $\mu^{-} / \mu^{+}$(the ratio of the motilities)[1].

\section{Conclusions}

All the experimental measurements of the sphere-to- plane geometry have shown a good agreement with those refer to point-to-plane and point-to-ring systems. The results achieved in the present work can be summarized as follows:

A geometrical configuration of sphere-to-plane electrodes has been devoted for positive and negative corona discharge at different inter-electrode separation.

The configuration of sphere-to-plane geometry is chose in order to provide different point sources of ions in air depending on the curvature of the sphere in front of the plane electrode.

The current and voltage data were acquired by data acquisition system that has been built experimentally for the present work.

The study concerned only with the influence of interelectrode separation on the current-voltage characteristics for both polarities.

The results achieved are independent of the diameter of the sphere.

The corona current decreased as the gap separation between the electrode increased and the corona inception voltage $V_{o}$ goes up for negative corona more than that for positive corona as the gap distance $\mathrm{S}$ increased.

An upward curvature was observed in current-voltage characteristics at lower applied voltage where the current itself is not very stable.

The upward trend in the characteristics of the acquired current and voltage was explained by an increasing participation of the electronic component on the total corona current.

The corona inception voltage tends to have a nonlinear behavior with the electrode gap distances and showed a good agreement with Xiangbo [4].the value of the power exponent $\mathrm{m}$ in this study was 0.41 and 0.54 for positive and negative corona respectively. The achieved values of $\mathrm{m}$ showed a good agreement with that referred by equation 3 .

The dimensional constant A was found to be inversely proportional to the electrode gap distance as $\mathrm{A} \propto \mathrm{S}^{-1.54}$ for positive corona, while it was $A \propto \mathrm{S}^{-2.15}$ for negative corona.

It was found that dimensional constants $\mathrm{K}^{+}$and $\mathrm{K}^{-}$ followed the same behavior of A but in a different value of power exponent as $\mathrm{K}^{+} \propto \mathrm{S}^{-1.57}$ for positive corona and $\mathrm{K}^{-} \propto \mathrm{S}^{-2.25}$ for negative corona.

The relationship of Townsend and Ferreira are held for both polarities at gap distance $(\mathrm{S} \geq 2.5 \mathrm{~cm})$ because the ratios $\mathrm{A}^{-} / \mathrm{A}^{+}$and $\mathrm{K}^{-} / \mathrm{K}^{+}$approached a constant value at these distances.

According to the above conclusions, similar common features between sphere-to-plane geometry and pointto-plane and point-to-ring systems have been found. 


\section{Acknowledgments}

The authors would like to thank Dr. Ahmed A. Tahir, the head of the department of computer science, for hishelp and useful comments.

\section{References}

[1] G. F. Leal Ferreira, O.N. Oliverira, Jr., and J. A. Giacometti, "Point-to-plane corona: Current-voltage characteristics for positive and negative polarity with evidence of an electronic component", J. Appl. Phys. Vol.59, No. 9, pp. 3045-3049, May 1986.

[2] J. Chen, "Direct current corona-enhanced chemical reactions", Ph.D Thesis, University of Minnesota, USA, 2002.

[3] B. L. Henson, “ A space-charge region model for microscopic steady coronas from points", J. Appl. Phys., Vol. 52, pp. 709-715, 1981.

[4] XiangboMeng, Hui Zhang and Jingxu (Jesse) Zhu, "A general empirical formula of current-voltage characteristics for point-to-plane geometry corona discharges", J
Phys. D: Appl. Phys. Vol. 41, pp. 065209 (10pp), 2008.

[5] K. Yamada, "An empirical formula for negative corona discharge current in point-grid electrode geometry" J. Appl. Phys, Vol. 96, pp. 2472-5, 2004.

[6] Pierluigi Giubbilini, "The current-voltage characteristics of point-to-plane corona", J. Appl. Phys., Vol. 64, No. 7, pp. 3730-3732, Oct. 1988.

[7] G. R. Gumurthy, "Corona between points plane electrodes in air at atmospheric pressure", IEEE Annual Report Conference on Electrical Insulation and Dielectric Phenomena, pp. 305, 2003.

[8] R. S. Sigmond, "Simple approximate treatment of unipolar space charge dominated coronas: The Warburg law and the saturation current", J. Appl. Phys., Vol. 53, pp. 891-898, 1982.

[9] M. Abdel-Salam, A. Ahmed, and A. Nasr EL-deen, "Inception Voltage of Corona Discharge from Suspended, Grounded and Stressed Particles in Uniform-Field GasInsulated-Gaps at Varying Pressures", International Journal of Plasma Environmental Science \& Technology, Vol.4, No.1, March 2010. 